\title{
Associations of Aminotransferases with Adverse Outcomes after Acute Ischemic Stroke: Results from China National Stroke Registry
}

\section{Lixia Zong}

Beijing Tiantan Hospital

\section{Xianwei Wang}

Beijing Tiantan Hospital

\section{Zixiao Li}

Beijing Tiantan Hospital

\section{Xingquan Zhao}

Beijing Tiantan Hospital

\section{Liping Liu}

Beijing Tiantan Hospital

Hao Li

Beijing Tiantan Hospital

Xia Meng

Beijing Tiantan Hospital

Yilong Wang

Beijing Tiantan Hospital

Yong-Jun Wang ( $\nabla$ yongjunwang@ncrend.org.cn )

Beijing Tiantan Hospital

\section{Research article}

Keywords: Aminotransferases, Ischemic stroke, Recurrent event, Mortality, Functional outcome

Posted Date: March 26th, 2020

DOI: https://doi.org/10.21203/rs.3.rs-18585/v1

License: (c) (1) This work is licensed under a Creative Commons Attribution 4.0 International License. Read Full License 


\section{Abstract}

Background The relationship between aminotransferases and cardiovascular outcomes has been inconsistent in previous studies. We aimed to investigate the association of aminotransferases with clinical outcomes after acute ischemic stroke (AIS) or transient ischemic attack (TIA).

Methods 17,178 AIS or TIA patients with serum alanine aminotransferase (ALT) levels $<120 \mathrm{U} / \mathrm{L}$ were included from the China National Stroke Registry (CNSR) for current analysis. Composite endpoint is comprised of recurrent stroke and all-cause mortality. Poor functional outcome is defined as modified Rankin scale of 3-6. Multivariable logistic regression was used to evaluate the risks of one-year all-cause mortality, recurrent stroke, composite endpoint and poor functional outcome according to increasing sexspecific quintiles of ALT/ aspartate aminotransferase (AST) respectively.

Results One-year incidences of all-cause mortality, recurrent stroke, composite endpoint and poor functional outcome were $11.9 \%, 6.0 \%, 13.7 \%$ and $28.2 \%$ respectively in patients with the lowest quintile of ALT, and $7.4 \%, 3.6 \%, 9.0 \%$ and $17.9 \%$ respectively in the highest quintile. Compared with the lowest ALT quintile, the adjusted odds ratios with $95 \%$ confidence interval of the highest quintile were $0.55(0.43-$ $0.70)$ for all-cause mortality, $0.61(0.45-0.83)$ for stroke recurrence, 0.62 (0.49-0.77) for composite endpoint, and 0.67 (0.56-0.80) for poor functional outcome. There was no significant interaction of ALT with age, sex, diabetes, dyslipidemia and alcohol consumption for all outcomes ( $p$ for interaction $\geq 0.10$ ).

Conclusions Low serum ALT may serve as an independent predictor for all-cause mortality, stroke recurrence, composite endpoint and poor functional outcome after stroke.

\section{Background}

Alanine aminotransferase (ALT) and aspartate aminotransferase (AST), abundantly present within hepatocytes, are enzymes catalyzing transfer of amino groups to generate products in gluconeogenesis and amino acid metabolism. Serum aminotransferase activities are routinely tested in clinical practice and commonly used as markers of liver health. In recent years, an increasing number of studies have suggested strong associations of serum aminotransferases, especially ALT with metabolic syndrome, insulin resistance and incident diabetes in the general population. ${ }^{1-3}$ So it is plausible that serum ALT has an association with cardiovascular disease (CVD).

However, findings concerning the relationship between ALT and CVD are inconsistent. ${ }^{4-10}$ While associations of elevated ALT with increased risk of CVD and mortality have been reported in the general population, $4,5,11,12$ there are also several studies showing significant associations between elevated ALT

and reduced CVD risk and mortality. 7, 9, 10,13 Two recent meta-analyses even suggested geographical variations in the association of ALT with CVD and mortality, as there was an inverse association for North American populations but a positive association for Asian populations. ${ }^{14,15}$ To date, there is limited data on the associations between ALT and CVD outcomes in secondary prevention populations. Therefore, 
based on China National Stroke Registry (CNSR), we investigated the association between serum ALT and adverse clinical outcomes after ischemic strokes or transient ischemic attack (TIA).

\section{Methods}

\section{Study Participants}

This study was conducted on the basis of CNSR II, which was a multicenter, hospital-based, prospective cohort study initiated in 2012 to evaluate the quality of care for hospitalized patients with cerebrovascular disease and to identify areas that need further improvement as compared with CNSR I performed in 2007. ${ }^{16}$ Patients were eligible for enrollment if they were of 18 years of age or older and hospitalized for a diagnosis of ischemic stroke, TIA, spontaneous intracerebral hemorrhage (ICH), or subarachnoid hemorrhage (SAH) within 7 days after onset. A total of 25,018 patients were continuously enrolled in the registry from 2012 to 2013 . For the current analysis, we excluded 3,426 (13.7\%) patients with intracerebral hemorrhage or subarachnoid hemorrhage, $167(0.7 \%)$ patients with self-reported liver disease, 1,704 (6.8\%) patients with missing ALT levels or ALT levels greater than three times the conventional upper limit of normal (120 U/L), and 2,543 (10.2\%) patients who were lost during the oneyear follow up. Finally, 17,178 patients were included in the present study (Fig. 1). The CNSR II study was approved by the central institutional review board at Beijing Tiantan hospital and written informed consent was obtained from all participants or their legally authorized representatives.

\section{Baseline Data Collection}

Detailed information regarding patient demographics, vascular risk factors, laboratory test, diagnosis, medicine use, stroke severity and complications were collected by trained research coordinators at each study center. Vascular risk factors included history of stroke, hypertension, diabetes, dyslipidemia, atrial fibrillation, coronary heart disease, previous or current smoking, and moderate or heavy alcohol consumption ( $\geq 2$ standardized alcohol drinks per day). Hypertension was defined as self-reported history of hypertension, any use of antihypertensive drug continuously taken for more than 2 weeks before index cerebrovascular diseases, or new diagnosis of hypertension at discharge. Diabetes mellitus was defined as self-reported history of diabetes mellitus, any use of glucose-lowering drugs, or new diagnosis of diabetes at discharge. Dyslipidemia was defined as new diagnosis of dyslipidemia at discharge or self-reported history of dyslipidemia. Atrial fibrillation was defined as history of atrial fibrillation confirmed by $\geq 1$ ECG or presence of the arrhythmia during hospitalization.

\section{ALT and AST Measurement}

Serum activities of ALT and AST were measured using the standardized kinetic method on fasting morning serum samples at each study center, according to the recommendations of the International Federation of Clinical Chemistry and Laboratory Medicine (IFCC). ${ }^{17,18}$ Since the reference range and cut- 
off values for ALT are controversial and varied by sex, ${ }^{19,20}$ we divided serum levels of ALT into sexspecific quintiles respectively, instead of using a cut-off value to define abnormality.

\section{Outcome Assessment}

Follow-up was done via telephone by trained research coordinators who were blinded to patients' baseline clinical status. Patients were asked the standardized follow-up questions to collect outcome data at 12month after disease onset. Adverse clinical outcomes are defined as all-cause mortality, recurrent stroke, composite endpoint and poor functional outcome. Recurrent stroke includes ischemic stroke, intracranial hemorrhage and subarachnoid hemorrhage. Composite endpoint is comprised of recurrent stroke and allcause mortality. Poor functional outcome is defined as modified Rankin scale of 3-6 (mRS, score range from 0 [no symptoms] to 6 [death]).

\section{Statistical Analysis}

Baseline characteristics and clinical outcomes of patients grouped by sex-specific quintiles of ALT ( $<13$, 13-17, 17-22, 22-30, $\geq 30 \mathrm{U} / \mathrm{L}$ for men, <12, 12-15, 15-19, 19-27, $\geq 27 \mathrm{U} / \mathrm{L}$ for women) were compared using $\chi^{2}$ test or one-way analysis of variance (ANOVA) as appropriate. Categorical variables are presented as proportions, and continuous variables are expressed as mean with standard deviation (SD) or medians with interquartile ranges (IQR).

The association of ALT or AST with clinical outcomes was evaluated using a logistic regression model, with the first quintile of ALT or AST as a reference group. We adjusted the following covariates known to be associated with adverse stroke outcomes or ALT or AST levels: age, sex, hypertension, diabetes, dyslipidemia, history of stroke, coronary heart disease, atrial fibrillation, current or previous smoking, moderate or heavy alcohol, white blood cell count, drugs used during hospitalization (antihypertensive agents, lipid-lowering agents and hypoglycemic agents), baseline national institutes of health stroke scale, body mass index, mRS at discharge and pneumonia during hospitalization.

We also performed a series of stratified analyses by age $(\geq 65$ vs $<65)$, sex, diabetes, dyslipidemia and alcohol consumption. To examine effect modification by age, sex, diabetes, dyslipidemia and alcohol consumption, we used a post-estimation Wald test in multivariable-adjusted logistic model to get an omnibus P-value for interaction between ALT quintiles and variables of interest. Further, we examined the pattern of associations between ALT and risks of adverse stroke outcomes using logistic regression model with restricted cubic splines for ALT adjusting for covariates. The median serum ALT level (18 U/L) was treated as the reference and eight knots were placed at 8, 12, 16, 20, 24, 28, 32 and $36 \mathrm{U} / \mathrm{L}$ of ALT levels. All analyses were conducted with SAS version 9.3 software (SAS institute).

\section{Results}

\section{Baseline Characteristics}


Baseline characteristics are presented in Table 1 (see Additional file 1). Of the total 17,178 patients, the mean age was 64.8 years, and $37.1 \%$ were female. Compared with patients in the lowest quintile of ALT, patients in the highest quintile were younger, more likely to be male and a heavy drinker, have higher body mass index, have higher proportion of diabetes and dyslipidemia, and have higher serum levels of AST, triglyceride, low density lipoprotein cholesterol, fasting blood-glucose and white blood cells. In addition, patients in the highest ALT quintile were less likely to have history of stroke and coronary heart disease, pneumonia during hospitalization and functional disability at discharge than patients in the lowest quintile. 
Table 1

Baseline characteristics in patients by stratified by sex-specific quintiles of ALT levels ${ }^{\dagger}$.

\begin{tabular}{|c|c|c|c|c|c|c|c|}
\hline \multirow[t]{4}{*}{ Characteristics } & \multirow{4}{*}{$\begin{array}{l}\text { Overall } \\
(n= \\
17178)\end{array}$} & \multicolumn{5}{|c|}{ ALT quintiles $^{\dagger}, \mathrm{U} / \mathrm{L}$} & \multirow{4}{*}{$\begin{array}{l}P \\
\text { value }\end{array}$} \\
\hline & & Q1 & Q2 & Q3 & Q4 & Q5 & \\
\hline & & $(n=$ & $(n=$ & $(n=$ & $(n=$ & $(n=$ & \\
\hline & & 3339) & 3219) & 3455) & 3572) & 3593) & \\
\hline Age (SD), year & $\begin{array}{l}64.8 \\
(11.9)\end{array}$ & $\begin{array}{l}69.2 \\
(11.5)\end{array}$ & $\begin{array}{l}66.2 \\
(11.5)\end{array}$ & $\begin{array}{l}64.7 \\
(11.6)\end{array}$ & $\begin{array}{l}63.5 \\
(11.3)\end{array}$ & $\begin{array}{l}60.9 \\
(12.0)\end{array}$ & $<0.001$ \\
\hline Female, n (\%) & $\begin{array}{l}6368 \\
(37.1)\end{array}$ & $\begin{array}{l}1270 \\
(38.0)\end{array}$ & $\begin{array}{l}1110 \\
(34.5)\end{array}$ & $\begin{array}{l}1245 \\
(36.0)\end{array}$ & $\begin{array}{l}1441 \\
(40.3)\end{array}$ & $\begin{array}{l}1302 \\
(36.2)\end{array}$ & $\begin{array}{l}< \\
0.001\end{array}$ \\
\hline \multicolumn{8}{|l|}{ Risk factors, n (\%) } \\
\hline Previous stroke & $\begin{array}{l}6169 \\
(35.9)\end{array}$ & $\begin{array}{l}1267 \\
(37.9)\end{array}$ & $\begin{array}{l}1157 \\
(35.9)\end{array}$ & $\begin{array}{l}1230 \\
(35.6)\end{array}$ & $\begin{array}{l}1269 \\
(35.5)\end{array}$ & $\begin{array}{l}1246 \\
(34.7)\end{array}$ & 0.07 \\
\hline Hypertension & $\begin{array}{l}12936 \\
(75.3)\end{array}$ & $\begin{array}{l}2509 \\
(75.1)\end{array}$ & $\begin{array}{l}2411 \\
(74.9)\end{array}$ & $\begin{array}{l}2578 \\
(74.6)\end{array}$ & $\begin{array}{l}2667 \\
(74.7)\end{array}$ & $\begin{array}{l}2771 \\
(77.1)\end{array}$ & 0.08 \\
\hline Diabetes & $\begin{array}{l}4341 \\
(25.3)\end{array}$ & $\begin{array}{l}757 \\
(22.7)\end{array}$ & $\begin{array}{l}765 \\
(23.8)\end{array}$ & $\begin{array}{l}892 \\
(25.8)\end{array}$ & $\begin{array}{l}915 \\
(25.6)\end{array}$ & $\begin{array}{l}1012 \\
(28.2)\end{array}$ & $\begin{array}{l}< \\
0.001\end{array}$ \\
\hline Dyslipidemia & $\begin{array}{l}5764 \\
(33.6)\end{array}$ & $\begin{array}{l}994 \\
(29.8)\end{array}$ & $\begin{array}{l}979 \\
(30.4)\end{array}$ & $\begin{array}{l}1202 \\
(34.8)\end{array}$ & $\begin{array}{l}1219 \\
(34.1)\end{array}$ & $\begin{array}{l}1370 \\
(38.1)\end{array}$ & $<0.001$ \\
\hline Atrial fibrillation & $\begin{array}{l}1173 \\
(6.8)\end{array}$ & $\begin{array}{l}254 \\
(7.6)\end{array}$ & $\begin{array}{l}205 \\
(6.4)\end{array}$ & $\begin{array}{l}246 \\
(7.1)\end{array}$ & $239(6.7)$ & $229(6.4)$ & 0.2 \\
\hline CHD & $\begin{array}{l}2339 \\
(13.6)\end{array}$ & $\begin{array}{l}503 \\
(15.1)\end{array}$ & $\begin{array}{l}447 \\
(13.9)\end{array}$ & $\begin{array}{l}480 \\
(13.9)\end{array}$ & $\begin{array}{l}489 \\
(13.7)\end{array}$ & $\begin{array}{l}420 \\
(11.7)\end{array}$ & $<.01$ \\
\hline $\begin{array}{l}\text { Current or } \\
\text { previous smoking }\end{array}$ & $\begin{array}{l}7483 \\
(43.6)\end{array}$ & $\begin{array}{l}1435 \\
(43.0)\end{array}$ & $\begin{array}{l}1468 \\
(45.6)\end{array}$ & $\begin{array}{l}1507 \\
(43.6)\end{array}$ & $\begin{array}{l}1490 \\
(41.7)\end{array}$ & $\begin{array}{l}1583 \\
(44.1)\end{array}$ & 0.02 \\
\hline $\begin{array}{l}\text { Moderate to } \\
\text { heavy alcohol, } \mathrm{n} \\
(\%)\end{array}$ & $\begin{array}{l}4753 \\
(27.7)\end{array}$ & $\begin{array}{l}859 \\
(25.7)\end{array}$ & $\begin{array}{l}901 \\
(28.0)\end{array}$ & $\begin{array}{l}931 \\
(26.9)\end{array}$ & $\begin{array}{l}989 \\
(27.7)\end{array}$ & $\begin{array}{l}1073 \\
(29.9)\end{array}$ & $<0.01$ \\
\hline $\begin{array}{l}\text { AST, U/L, median } \\
\text { (IQR) }\end{array}$ & $\begin{array}{l}21.0 \\
(17.0- \\
27.0)\end{array}$ & $\begin{array}{l}16.0 \\
(14.0- \\
19.8)\end{array}$ & $\begin{array}{l}18.0 \\
(15.0- \\
21.0)\end{array}$ & $\begin{array}{l}20.0 \\
(17.0- \\
24.0)\end{array}$ & $\begin{array}{l}23.0 \\
(19.0- \\
27.0)\end{array}$ & $\begin{array}{l}30.5 \\
(24.0- \\
40.0)\end{array}$ & $<0.001$ \\
\hline
\end{tabular}

ALT, alanine aminotransferase; AST, aspartate aminotransferase; TG, triglyceride; LDL-C, low density lipoprotein cholesterol; HDL-C, high density lipoprotein cholesterol; FBG, fasting blood-glucose; WBC, white blood cells; CHD, coronary heart disease; BMI, body mass index; NIHSS, national institutes of health stroke scale; mRS, modified Rankin score; SD, standard deviation; IQR, interquartile range; Q, quintile. ${ }^{\dagger}$ sex-specific cut-points for ALT per quintile are < 13[men], <12[women] for Q1; 13-17[men], 12-15[women] for Q2; 17-22[men], 15-19[women] for Q3; 22-30[men], 19-27[women] for Q4; $\geq 30$ [men], $\geq 27$ [women] for Q5. 


\begin{tabular}{|c|c|c|c|c|c|c|c|}
\hline \multirow[t]{4}{*}{ Characteristics } & \multirow{4}{*}{$\begin{array}{l}\text { Overall } \\
(n= \\
17178)\end{array}$} & \multicolumn{5}{|c|}{ ALT quintiles ${ }^{\dagger}, \mathrm{U} / \mathrm{L}$} & \multirow{4}{*}{$\begin{array}{l}\mathrm{P} \\
\text { value }\end{array}$} \\
\hline & & Q1 & Q2 & Q3 & Q4 & Q5 & \\
\hline & & $(n=$ & $(n=$ & $(n=$ & $(n=$ & $(n=$ & \\
\hline & & 3339) & 3219) & 3455) & 3572) & 3593) & \\
\hline $\begin{array}{l}\mathrm{TG}, \mathrm{mg} / \mathrm{dL} \\
\text { median (IQR) }\end{array}$ & $\begin{array}{l}118.6 \\
(85.8- \\
172.6)\end{array}$ & $\begin{array}{l}105.3 \\
(76.1- \\
142.5)\end{array}$ & $\begin{array}{l}112.4 \\
(80.5- \\
154.9)\end{array}$ & $\begin{array}{l}118.6 \\
(86.7- \\
170.8)\end{array}$ & $\begin{array}{l}125.7 \\
(91.2- \\
185.8)\end{array}$ & $\begin{array}{l}139.8 \\
(99.1- \\
203.5)\end{array}$ & $\begin{array}{l}< \\
0.001\end{array}$ \\
\hline $\begin{array}{l}\mathrm{LDL}-\mathrm{C}, \mathrm{mg} / \mathrm{dL} \text {, } \\
\text { median (IQR) }\end{array}$ & $\begin{array}{l}105.8 \\
(85.3- \\
127.4)\end{array}$ & $\begin{array}{l}104.3 \\
(83.4- \\
125.5)\end{array}$ & $\begin{array}{l}105.4 \\
(85.3- \\
125.1)\end{array}$ & $\begin{array}{l}105.8 \\
(86.5- \\
128.2)\end{array}$ & $\begin{array}{l}106.0 \\
(86.1- \\
129.0)\end{array}$ & $\begin{array}{l}106.2 \\
(85.7- \\
129.7)\end{array}$ & $\begin{array}{l}< \\
0.001\end{array}$ \\
\hline $\begin{array}{l}\mathrm{HDL}-\mathrm{C}, \mathrm{mg} / \mathrm{dL} \text {, } \\
\text { median (IQR) }\end{array}$ & $\begin{array}{l}44.2 \\
(37.2- \\
52.7)\end{array}$ & $\begin{array}{l}44.6 \\
(37.2- \\
53.1)\end{array}$ & $\begin{array}{l}44.2 \\
(38.0- \\
53.1)\end{array}$ & $\begin{array}{l}44.2 \\
(37.6- \\
52.7)\end{array}$ & $\begin{array}{l}44.2 \\
(37.2- \\
52.3)\end{array}$ & $\begin{array}{l}43.8 \\
(36.8- \\
51.6)\end{array}$ & 0.09 \\
\hline $\begin{array}{l}\mathrm{FBG}, \mathrm{mmol} / \mathrm{L} \\
\text { median (IQR) }\end{array}$ & $\begin{array}{l}5.5 \\
(4.8- \\
6.7)\end{array}$ & $\begin{array}{l}5.3 \\
(4.7- \\
6.4)\end{array}$ & $\begin{array}{l}5.3 \\
(4.8- \\
6.5)\end{array}$ & $\begin{array}{l}5.4 \\
(4.8- \\
6.6)\end{array}$ & $\begin{array}{l}5.6(4.9- \\
6.8)\end{array}$ & $\begin{array}{l}5.7(5.0- \\
7.2)\end{array}$ & $\begin{array}{l}<.001 \\
0.001\end{array}$ \\
\hline $\begin{array}{l}\mathrm{WBC}, 10^{9} / \mathrm{L} \\
\text { median (IQR) }\end{array}$ & $\begin{array}{l}6.7 \\
(5.5- \\
8.3)\end{array}$ & $\begin{array}{l}6.6 \\
(5.4- \\
8.1)\end{array}$ & $\begin{array}{l}6.6 \\
(5.5- \\
8.1)\end{array}$ & $\begin{array}{l}6.7 \\
(5.5- \\
8.3)\end{array}$ & $\begin{array}{l}6.8(5.6- \\
8.4)\end{array}$ & $\begin{array}{l}6.9(5.7- \\
8.5)\end{array}$ & $\dot{0.001}$ \\
\hline $\begin{array}{l}\mathrm{BMI}, \mathrm{kg} / \mathrm{m}^{2} \\
\text { median (IQR) }\end{array}$ & $\begin{array}{l}24.0 \\
(22.0- \\
25.7)\end{array}$ & $\begin{array}{l}23.4 \\
(21.3- \\
24.9)\end{array}$ & $\begin{array}{l}23.7 \\
(21.8- \\
25.4)\end{array}$ & $\begin{array}{l}23.9 \\
(22.0- \\
25.7)\end{array}$ & $\begin{array}{l}24.2 \\
(22.5- \\
26.1)\end{array}$ & $\begin{array}{l}24.2 \\
(22.6- \\
26.4)\end{array}$ & $\dot{0} 001$ \\
\hline $\begin{array}{l}\text { NIHSS at } \\
\text { admission, } \\
\text { median (IQR) }\end{array}$ & $3(1-6)$ & $4(1-6)$ & $3(1-6)$ & $3(1-7)$ & $3(1-6)$ & $3(1-6)$ & 0.12 \\
\hline \multicolumn{8}{|l|}{$\mathrm{mRS}$ at discharge } \\
\hline $0-2$ & $\begin{array}{l}13320 \\
(78.4)\end{array}$ & $\begin{array}{l}2486 \\
(75.3)\end{array}$ & $\begin{array}{l}2502 \\
(78.6)\end{array}$ & $\begin{array}{l}2697 \\
(78.8)\end{array}$ & $\begin{array}{l}2820 \\
(79.7)\end{array}$ & $\begin{array}{l}2815 \\
(79.6)\end{array}$ & \multirow[t]{2}{*}{$<.001$} \\
\hline $3-5$ & $\begin{array}{l}3660 \\
(21.6)\end{array}$ & $\begin{array}{l}814 \\
(24.7)\end{array}$ & $\begin{array}{l}681 \\
(21.4)\end{array}$ & $\begin{array}{l}726 \\
(21.2)\end{array}$ & $\begin{array}{l}717 \\
(20.3)\end{array}$ & $\begin{array}{l}722 \\
(20.4)\end{array}$ & \\
\hline \multicolumn{8}{|l|}{$\begin{array}{l}\text { Medication } \\
\text { during } \\
\text { hospitalization }\end{array}$} \\
\hline $\begin{array}{l}\text { Antihypertensive } \\
\text { drugs }\end{array}$ & $\begin{array}{l}8352 \\
(48.6)\end{array}$ & $\begin{array}{l}1585 \\
(47.5)\end{array}$ & $\begin{array}{l}1571 \\
(48.8)\end{array}$ & $\begin{array}{l}1681 \\
(48.7)\end{array}$ & $\begin{array}{l}1743 \\
(48.8)\end{array}$ & $\begin{array}{l}1772 \\
(49.3)\end{array}$ & 0.63 \\
\hline \multicolumn{8}{|c|}{$\begin{array}{l}\text { ALT, alanine aminotransferase; AST, aspartate aminotransferase; TG, triglyceride; LDL-C, low density } \\
\text { lipopprotein cholesterol; HDL-C, high density lipoprotein cholesterol; FBG, fasting blood-glucose; WBC, } \\
\text { white blood cells; CHD, coronary heart disease; BMl, body mass index; NIHSS, national institutes of } \\
\text { health stroke scale; mRS, modified Rankin score; SD, standard deviation; IQR, interquartile range; Q, } \\
\text { quintile. }{ }^{\dagger} \text { sex-specific cut-points for ALT per quintile are < } 13 \text { [men], <12[women] for Q1; 13-17[men], } \\
12-15 \text { [women] for Q2; } 17-22 \text { [men], 15-19[women] for Q3; 22-30[men], 19-27[women] for Q4; } \\
\geq 30 \text { [men], } \geq 27 \text { [women] for Q5. }\end{array}$} \\
\hline
\end{tabular}




\begin{tabular}{|c|c|c|c|c|c|c|c|}
\hline \multirow[t]{4}{*}{ Characteristics } & \multirow{4}{*}{$\begin{array}{l}\begin{array}{l}\text { Overall } \\
(n=\end{array} \\
17178)\end{array}$} & \multicolumn{5}{|c|}{ ALT quintiles $^{\dagger}, \mathrm{U} / \mathrm{L}$} & \multirow{4}{*}{$\begin{array}{l}P \\
\text { value }\end{array}$} \\
\hline & & Q1 & Q2 & Q3 & Q4 & Q5 & \\
\hline & & $(n=$ & $(n=$ & $(n=$ & $(n=$ & $(n=$ & \\
\hline & & 3339) & 3219) & $3455)$ & 3572) & 3593) & \\
\hline $\begin{array}{l}\text { Hypoglycemia } \\
\text { drug }\end{array}$ & $\begin{array}{l}3386 \\
(19.7)\end{array}$ & $\begin{array}{l}578 \\
(17.3)\end{array}$ & $\begin{array}{l}609 \\
(18.9)\end{array}$ & $\begin{array}{l}675 \\
(19.5)\end{array}$ & $\begin{array}{l}714 \\
(20.0)\end{array}$ & $\begin{array}{l}810 \\
(22.5)\end{array}$ & $\begin{array}{l}< \\
0.001\end{array}$ \\
\hline $\begin{array}{l}\text { Lipid-lowering } \\
\text { drugs }\end{array}$ & $\begin{array}{l}8843 \\
(51.5)\end{array}$ & $\begin{array}{l}1561 \\
(46.8)\end{array}$ & $\begin{array}{l}1604 \\
(49.8)\end{array}$ & $\begin{array}{l}1812 \\
(52.4)\end{array}$ & $\begin{array}{l}1926 \\
(53.9)\end{array}$ & $\begin{array}{l}1940 \\
(54.0)\end{array}$ & $<.001$ \\
\hline $\begin{array}{l}\text { Pneumonia } \\
\text { during } \\
\text { hospitalization }\end{array}$ & $\begin{array}{l}1203 \\
(7.0)\end{array}$ & $\begin{array}{l}304 \\
(9.1)\end{array}$ & $\begin{array}{l}221 \\
(6.9)\end{array}$ & $\begin{array}{l}226 \\
(6.5)\end{array}$ & $218(6.1)$ & $234(6.5)$ & $<.001$ \\
\hline \multicolumn{8}{|c|}{$\begin{array}{l}\text { ALT, alanine aminotransferase; AST, aspartate aminotransferase; TG, triglyceride; LDL-C, low density } \\
\text { lipoprotein cholesterol; HDL-C, high density lipoprotein cholesterol; FBG, fasting blood-glucose; WBC, } \\
\text { white blood cells; CHD, coronary heart disease; BMI, body mass index; NIHSS, national institutes of } \\
\text { health stroke scale; mRS, modified Rankin score; SD, standard deviation; IQR, interquartile range; Q, } \\
\text { quintile. }{ }^{\dagger} \text { sex-specific cut-points for ALT per quintile are }<13 \text { [men], < } 12 \text { [women] for Q1; } 13-17 \text { [men], } \\
12-15[\text { [women] for Q2; } 17-22 \text { [men], 15-19[women] for Q3; } 22-30 \text { [men], 19-27[women] for Q4; } \\
\geq 30 \text { [men], } \geq 27 \text { [women] for Q5. }\end{array}$} \\
\hline
\end{tabular}

\section{One-year outcomes among patients grouped by ALT quintiles}

As shown in Table 2, the one-year incidences of all clinical outcomes decreased as ALT quintiles increased ( $p<0.001$ for all outcomes). The incidence rates of all-cause mortality, recurrent stroke, composite endpoint and poor functional outcome in the first quintile of ALT were $11.9 \%, 6.0 \%, 13.7 \%$ and $28.2 \%$ respectively; and in the fifth quintile,they were $7.4 \%, 3.6 \%, 9.0 \%$ and $17.9 \%$ respectively. 
Table 2

Rates of one-year outcomes according to quintiles of ALT levels.

\begin{tabular}{|c|c|c|c|c|c|c|c|}
\hline \multirow[t]{2}{*}{ Outcomes } & \multirow[t]{2}{*}{ Overall } & \multicolumn{5}{|c|}{ ALT quintiles } & \multirow{2}{*}{$\begin{array}{l}P \\
\text { value }\end{array}$} \\
\hline & & Q1 & Q2 & Q3 & Q4 & Q5 & \\
\hline $\begin{array}{l}\text { All-cause mortality, } \mathrm{n} \\
(\%)\end{array}$ & $\begin{array}{l}1488 \\
(8.7)\end{array}$ & $\begin{array}{l}397 \\
(11.9)\end{array}$ & $\begin{array}{l}255 \\
(7.9)\end{array}$ & $\begin{array}{l}301 \\
(8.7)\end{array}$ & $\begin{array}{l}268 \\
(7.5)\end{array}$ & $\begin{array}{l}267 \\
(7.4)\end{array}$ & $\begin{array}{l}< \\
0.001\end{array}$ \\
\hline Recurrent stroke, n (\%) & $\begin{array}{l}723 \\
(4.5)\end{array}$ & $\begin{array}{l}182 \\
(6.0)\end{array}$ & $\begin{array}{l}124 \\
(4.2)\end{array}$ & $\begin{array}{l}159 \\
(4.9)\end{array}$ & $\begin{array}{l}137 \\
(4.1)\end{array}$ & $\begin{array}{l}121 \\
(3.6)\end{array}$ & $\begin{array}{l}< \\
0.001\end{array}$ \\
\hline $\begin{array}{l}\text { Composite endpoint, } n \\
\text { (\%) }\end{array}$ & $\begin{array}{l}1786 \\
(10.4)\end{array}$ & $\begin{array}{l}459 \\
(13.7)\end{array}$ & $\begin{array}{l}317 \\
(9.8)\end{array}$ & $\begin{array}{l}364 \\
(10.5)\end{array}$ & $\begin{array}{l}323 \\
(9.0)\end{array}$ & $\begin{array}{l}323 \\
(9.0)\end{array}$ & $\dot{0} 001$ \\
\hline $\begin{array}{l}\text { Poor functional } \\
\text { outcome, } \mathrm{n}(\%)\end{array}$ & $\begin{array}{l}3661 \\
(21.3)\end{array}$ & $\begin{array}{l}940 \\
(28.2)\end{array}$ & $\begin{array}{l}652 \\
(20.3)\end{array}$ & $\begin{array}{l}736 \\
(21.3)\end{array}$ & $\begin{array}{l}689 \\
(19.3)\end{array}$ & $\begin{array}{l}644 \\
(17.9)\end{array}$ & $<.001$ \\
\hline
\end{tabular}

\section{Aminotransferase and Adverse Clinical Outcomes after stroke}

Data on associations between ALT levels and adverse clinical outcomes are presented in Fig. 2. In the multiple logistic regression analysis, for all the adverse clinical outcomes, the adjusted odds ratios for the second to fifth ALT quintiles versus the first quintile were all below 1.00 ( $p$ for trend $<0.01$ ). Compared with the first quintile of ALT, the adjusted odds ratio with $95 \%$ confidence interval of the fifth quintile was $0.55(0.43-0.70)$ for all-cause mortality, $0.61(0.45-0.83)$ for recurrent stroke, $0.62(0.49-0.77)$ for composite endpoint and $0.67(0.56-0.80)$ for poor functional outcome, respectively.

The patterns of associations between ALT and clinical outcomes are depicted in Fig. 3. Overall, ALT (especially within the normal range) displayed a significant inverse association with adverse clinical outcomes. In subgroup analysis (Supplemental table, Please see Additional file 2), there was no evidence of interaction of age ( $<65 \mathrm{vs} \geq 65$ ), sex, diabetes, dyslipidemia and alcohol consumption with ALT for all the adverse clinical outcomes ( $p$ values of interaction $\geq 0.05$ for all outcomes).

Elevated AST levels was associated with increased risk of all-cause mortality and composite endpoint (Table 3, Please see Additional file 3). Compared with the first quintile of AST, the adjusted odds ratio with $95 \%$ confidence interval of the fifth quintile was $1.81(1.42-2.30)$ for all-cause mortality and $1.48(1.20-$ 1.83) for composite endpoint. No significant association was observed between AST levels and recurrent stroke or poor functional outcome. 
Table 3

Adjusted odds ratios of AST levels for one-year adverse outcomes.

\begin{tabular}{|c|c|c|c|c|c|}
\hline \multirow[t]{2}{*}{ Outcomes } & \multicolumn{5}{|c|}{ Adjusted OR (95\% Cl) } \\
\hline & Q1 & Q2 & Q3 & Q4 & Q5 \\
\hline All-cause mortality & $\begin{array}{l}1.00 \\
\text { (Reference) }\end{array}$ & $\begin{array}{l}1.01(0.81- \\
1.26)\end{array}$ & $\begin{array}{l}1.01(0.81- \\
1.26)\end{array}$ & $\begin{array}{l}1.18(0.93- \\
1.48)\end{array}$ & $\begin{array}{l}1.81(1.42- \\
2.30)\end{array}$ \\
\hline Recurrent stroke & $\begin{array}{l}1.00 \\
\text { (Reference) }\end{array}$ & $\begin{array}{l}0.86(0.66- \\
1.12)\end{array}$ & $\begin{array}{l}0.89(0.68- \\
1.15)\end{array}$ & $\begin{array}{l}1.05(0.79- \\
1.38)\end{array}$ & $\begin{array}{l}1.21(0.90- \\
1.62)\end{array}$ \\
\hline $\begin{array}{l}\text { Composite } \\
\text { endpoint }\end{array}$ & $\begin{array}{l}1.00 \\
\text { (Reference) }\end{array}$ & $\begin{array}{l}0.90(0.74- \\
1.09)\end{array}$ & $\begin{array}{l}0.93(0.77- \\
1.12)\end{array}$ & $\begin{array}{l}1.07(0.88- \\
1.31)\end{array}$ & $\begin{array}{l}1.48(1.20- \\
1.83)\end{array}$ \\
\hline $\begin{array}{l}\text { Poor functional } \\
\text { outcome }\end{array}$ & $\begin{array}{l}1.00 \\
\text { (Reference) }\end{array}$ & $\begin{array}{l}0.93(0.80- \\
1.09)\end{array}$ & $\begin{array}{l}0.89(0.77- \\
1.04)\end{array}$ & $\begin{array}{l}1.03(0.87- \\
1.21)\end{array}$ & $\begin{array}{l}1.16(0.97- \\
1.39)\end{array}$ \\
\hline \multicolumn{6}{|c|}{$\begin{array}{l}\text { Values represent odds ratios with } 95 \% \text { confidential interval in multivariable analysis adjusting for age } \\
\text { gender, diabetes, hypertension, dyslipidemia, history of stroke, coronary heart disease, atrial } \\
\text { fibrillation, current or previous smoking, moderate or heavy alcohol, baseline National Institutes of } \\
\text { Health Stroke Scale (NIHSS), mRS at discharge, body mass index (BMI), ALT levels, white blood cell } \\
\text { count, antihypertensive drugs, lipid-lowering drugs, hypoglycemia drugs and pneumonia during } \\
\text { hospitalization. OR, odds ratio; Cl, confidence interval; Q, quintile. Cut-points for AST per quintile are < } \\
16 \text { for Q1; } 16-19 \text { for Q2; } 19-23 \text { for Q3; } 23-29 \text { for Q4; } \geq 29 \text { for Q5. }\end{array}$} \\
\hline
\end{tabular}

\section{Discussion}

In this large prospective cohort study, with ALT levels largely within the conventional normal range, we found that ALT levels were inversely associated with adverse clinical outcomes after acute ischemic stroke or TIA in Chinese patients, independent of traditional vascular risk factors and not significantly modified by age, sex, diabetes, dyslipidemia and alcohol consumption. The risks of one-year all-cause mortality, recurrent stroke, composite endpoint and poor functional outcomes in patients with higher ALT levels ( $\geq 30 \mathrm{U} / \mathrm{L}$ for men and $\geq 27 \mathrm{U} / \mathrm{L}$ for women) decreased about $30 \%-45 \%$ as compared with patients with lower $A L T(<13 \mathrm{U} / \mathrm{L}$ for men and $<12 \mathrm{U} / \mathrm{L}$ for women). In addition, AST levels were positively associated with all-cause mortality and composite endpoint. Our findings are relevant and offer further insight concerning the relationship between aminotransferases and clinical outcomes after acute ischemic cerebrovascular diseases.

Elevated ALT has been associated with insulin resistance, metabolic syndrome, incident diabetes and other risk factors for atherosclerosis, ${ }^{1-3,21,22}$ so it seems paradoxical that elevated levels of ALT are associated with decreased risk of cardiovascular outcomes. However, our results are consistent with findings from several previous studies, which revealed inverse associations of ALT with cardiovascular risk and all-cause mortality in community-based populations and populations with cardiovascular risk factors. $7,9,10,13$ The mechanism behind the inverse associations between ALT and adverse cardiovascular outcomes are unclear. Nonetheless, some hypotheses have been proposed in prior 
studies. It was suggested that low ALT activity reflects reduced functionality and impaired synthetic capacity of hepatocytes, and as hepatocytes are important mediators in lipid metabolism, detoxification and glucose metabolism, a decrease in functional hepatocytes can be detrimental. 7, 13, 23, 24 Low ALT activity also reflects poor nutrition and reduced blood flow to the liver, which might increase the susceptibility to toxins and subsequently to diseases. ${ }^{25,26}$ In addition, low ALT activity is an indicator of aging and frailty, which are associated with an increased risk of CVD. ${ }^{25,27}$ There were limited data for us to explore the mechanism behind this association, however, our study is consistent with previous studies showing an inverse correlation of ALT with age. ${ }^{25}$

In contrast to our study, there were several studies that found no association or positive association between ALT and cardiovascular risk. ${ }^{1,4-6}$ The discrepancies in results may be attributed to differences in study populations, the range of ALT levels, and follow-up period. In particularly, ALT levels within the normal range were inversely associated with subsequent cardiovascular risks in some studies (including our study), 7, 9, 10,13 but ALT levels above the upper limit of normality were associated with increased risks for CVD, which might be in part due to underlying non-alcoholic fatty liver disease and its consequent effects on metabolic dysfunction and diabetes. ${ }^{4,28,29}$ Additionally, Kunutsor and colleagues addressed the modified effect of age on the association of ALT with cardiovascular risk, showing a significant inverse association in participants over 50 years but not among younger individuals. ${ }^{13}$ However, we didn't find the effect modification by age ( $<65$ vs $\geq 65$ ) on the relationship between ALT and adverse clinical outcomes in our population. This may be due to the relatively older age of the overall population (mean age, 65 years) in our study.

We also observed an inverse association between ALT and one-year poor functional outcomes after stroke, which is consistent with a previous study showing that high levels of ALT and AST were associated with good outcome at 3 months (mRS $0-2$ ) after acute ischemic stroke. ${ }^{30}$ The association between ALT and good functional outcomes can be presumably explained by the capacity of aminotransferases to metabolize blood glutamate and reduce the neurotoxic effect of elevated glutamate levels in the brain following ischemic stroke. ${ }^{30,31}$ However, we didn't find a significant association between AST and functional outcome. As the half-life of serum AST is about 17 hours, the time from stroke onset to blood drawn may affect the association between AST and clinical outcomes after stroke. The relationship between AST and cardiovascular outcomes has been quite inconsistent in prior studies and merits further exploration.

There are some limitations in the present study. First, serum ALT and AST levels were measured at each study site. However, the values of ALT and AST tested across the involved sites would be comparable, since the measurement procedures of ALT and AST in all sites were carried out according to the recommendation of IFCC (2011). Second, there was a lack of data on ultrasound, liver biopsies or imaging information for the diagnosis of non-alcoholic fatty liver disease, which could be a link between ALT or AST and CVD. Third, as we have excluded patients lacking ALT values or follow-up information, 
there might be selection bias. Finally, there might be some unmeasured or residual confounding effects due to the observational design of our study.

\section{Conclusions}

In conclusion, ALT levels were inversely associated with risk of all-cause mortality, stroke recurrence, composite endpoint and poor functional outcomes after ischemic stroke or TIA in this prospective study in China. Low serum ALT within normal range may serve as an independent predictor for clinical outcomes after acute ischemic cerebrovascular disease.

\section{Abbreviations}

AIS

acute ischemic stroke

ALT

alanine aminotransferase

ANOVA

one-way analysis of variance

AST

aspartate aminotransferase

CNSR

China National Stroke Registry

CVD

cardiovascular disease

$\mathrm{ICH}$

intracerebral hemorrhage

IFCC

International Federation of Clinical Chemistry and Laboratory Medicine

IQR

interquartile ranges

mRS

modified Rankin scale

SAH

subarachnoid hemorrhage

SD

standard deviation

TIA

transient ischemic attack

\section{Declarations}




\section{Ethics approval and consent to participate}

The CNSR II study was approved by the central institutional review board at Beijing Tiantan hospital and written informed consent was obtained from all participants or their legally authorized representatives.

\section{Consent for publication}

Not applicable.

\section{Availability of data and materials}

The datasets used and analysed during the current study are available from the corresponding author on reasonable request.

\section{Competing interests}

The authors declare that they have no competing interests.

\section{Funding}

This study was supported by grants from the Ministry of Science and Technology of the People's Republic of China (2012ZX09303 and 2011BAI08B02) and the National Key Technology Research and Development Program of China (2017YFC1310900 and 2017YFC1310901).

\section{Authors' contributions}

X.W.W., L.X.Z. and Y.J.W. contributed to the conception and design of this study; all authors contributed to acquisition and analysis of data and to critical revisions of the manuscript; X.W.W. and L.X.Z. drafted the manuscript and prepared the figures. All authors read and approved the final manuscript.

\section{Acknowledgments}

We thank all participating hospitals, their physicians and nurses, and the CNSR II Steering Committee members. 


\section{References}

1.

Goessling W, Massaro JM, Vasan RS, D'Agostino RB, Sr., Ellison RC, Fox CS. Aminotransferase levels and 20-year risk of metabolic syndrome, diabetes, and cardiovascular disease. Gastroenterology. 2008;135:1935-44. 1944 e1931.

2.

Schneider AL, Lazo M, Ndumele CE, Pankow JS, Coresh J, Clark JM, et al. Liver enzymes, race, gender and diabetes risk: The atherosclerosis risk in communities (aric) study. Diabetic medicine: a journal of the British Diabetic Association. 2013;30:926-33.

3.

Hanley AJ, Williams K, Festa A, Wagenknecht LE, D'Agostino RB Jr, Haffner SM. Liver markers and development of the metabolic syndrome: The insulin resistance atherosclerosis study. Diabetes. 2005;54:3140-7.

4.

Schindhelm RK, Dekker JM, Nijpels G, Bouter LM, Stehouwer CD, Heine RJ, et al. Alanine aminotransferase predicts coronary heart disease events: A 10-year follow-up of the hoorn study. Atherosclerosis. 2007;191:391-6.

5 .

Gao M, Cheng Y, Zheng Y, Zhang W, Wang L, Qin L. Association of serum transaminases with short- and long-term outcomes in patients with st-elevation myocardial infarction undergoing primary percutaneous coronary intervention. BMC Cardiovasc Disord. 2017;17:43.

6.

Monami M, Bardini G, Lamanna C, Pala L, Cresci B, Francesconi P, et al. Liver enzymes and risk of diabetes and cardiovascular disease: Results of the firenze bagno a ripoli (fibar) study. Metabolism. 2008;57:387-92.

7.

Ford I, Mooijaart SP, Lloyd S, Murray HM, Westendorp RG, de Craen AJ, et al. The inverse relationship between alanine aminotransferase in the normal range and adverse cardiovascular and noncardiovascular outcomes. Int J Epidemiol. 2011;40:1530-8.

8.

Ruhl CE, Everhart JE. The association of low serum alanine aminotransferase activity with mortality in the us population. Am J Epidemiol. 2013;178:1702-11.

9.

Williams KH, Sullivan DR, Veillard AS, O'Brien R, George J, Jenkins AJ, et al. Low alanine aminotransferase levels and higher number of cardiovascular events in people with type 2 diabetes: Analysis of the fenofibrate intervention and event lowering in diabetes (field) study. Diabetic medicine: a journal of the British Diabetic Association. 2016;33:356-64.

10. 
Harada PH, Cook NR, Cohen DE, Paynter NP, Rose L, Ridker PM. Relation of alanine aminotransferase levels to cardiovascular events and statin efficacy. Am J Cardiol. 2016;118:49-55.

11.

Kim HC, Kang DR, Nam CM, Hur NW, Shim JS, Jee SH, et al. Elevated serum aminotransferase level as a predictor of intracerebral hemorrhage: Korea medical insurance corporation study. Stroke. 2005;36:16427.

12.

Lee TH, Kim WR, Benson JT, Therneau TM, Melton LJ. 3rd. Serum aminotransferase activity and mortality risk in a united states community. Hepatology 2008;47:880-7.

13.

Kunutsor SK, Bakker SJ, Kootstra-Ros JE, Blokzijl H, Gansevoort RT, Dullaart RP. Inverse linear associations between liver aminotransferases and incident cardiovascular disease risk: The prevend study. Atherosclerosis. 2015;243:138-47.

14.

Kunutsor SK, Apekey TA, Seddoh D, Walley J. Liver enzymes and risk of all-cause mortality in general populations: A systematic review and meta-analysis. Int J Epidemiol. 2014;43:187-201. 15.

Kunutsor SK, Apekey TA, Khan H. Liver enzymes and risk of cardiovascular disease in the general population: A meta-analysis of prospective cohort studies. Atherosclerosis. 2014;236:7-17. 16.

Wang Y, Cui L, Ji X, Dong Q, Zeng J, Wang Y, et al. The china national stroke registry for patients with acute cerebrovascular events: Design, rationale, and baseline patient characteristics. Int J Stroke. 2011;6:355-61.

17.

Schumann G, Bonora R, Ceriotti F, Ferard G, Ferrero CA, Franck PF, et al. Ifcc primary reference procedures for the measurement of catalytic activity concentrations of enzymes at 37 degrees $\mathrm{c}$. International federation of clinical chemistry and laboratory medicine. Part 4. Reference procedure for the measurement of catalytic concentration of alanine aminotransferase. Clin Chem Lab Med. 2002;40:71824.

18.

Schumann G, Bonora R, Ceriotti F, Ferard G, Ferrero CA, Franck PF, et al. Ifcc primary reference procedures for the measurement of catalytic activity concentrations of enzymes at 37 degrees $c$. International federation of clinical chemistry and laboratory medicine. Part 5. Reference procedure for the measurement of catalytic concentration of aspartate aminotransferase. Clin Chem Lab Med. 2002;40:725-33.

19.

Ratziu V, Imbert-Bismut F, Messous D, Poynard T. The elusiveness of "normal" alt in fatty liver. Hepatology. 2004;39:1172.

20. 
Pacifico L, Ferraro F, Bonci E, Anania C, Romaggioli S, Chiesa C. Upper limit of normal for alanine aminotransferase: Quo vadis? Clin Chim Acta. 2013;422:29-39.

21.

Lorenzo C, Hanley AJ, Rewers MJ, Haffner SM. The association of alanine aminotransferase within the normal and mildly elevated range with lipoproteins and apolipoproteins: The insulin resistance atherosclerosis study. Diabetologia. 2013;56:746-57.

22.

Siddiqui MS, Sterling RK, Luketic VA, Puri P, Stravitz RT, Bouneva I, et al. Association between high-normal levels of alanine aminotransferase and risk factors for atherogenesis. Gastroenterology. 2013;145:12719.e1271-1273.

23.

Schooling CM, Kelvin EA, Jones HE. Alanine transaminase has opposite associations with death from diabetes and ischemic heart disease in nhanes iii. Ann Epidemiol. 2012;22:789-98.

24.

DG LEC, Cogger VC, McCuskey RS, Smedsrod RDEC, Sorensen B. KK, et al. Age-related changes in the liver sinusoidal endothelium: A mechanism for dyslipidemia. Ann N Y Acad Sci. 2007;1114:79-87. 25.

Dong MH, Bettencourt R, Brenner DA, Barrett-Connor E, Loomba R. Serum levels of alanine aminotransferase decrease with age in longitudinal analysis. Clin Gastroenterol Hepatol 2012;10:285-90 e281.

26.

Schmucker DL. Age-related changes in liver structure and function: Implications for disease ? Exp Gerontol. 2005;40:650-9.

27.

Le Couteur DG, Blyth FM, Creasey HM, Handelsman DJ, Naganathan V, Sambrook PN, et al. The association of alanine transaminase with aging, frailty, and mortality. J Gerontol A Biol Sci Med Sci. 2010;65:712-7.

28.

Targher G, Day CP, Bonora E. Risk of cardiovascular disease in patients with nonalcoholic fatty liver disease. N Engl J Med. 2010;363:1341-50.

29.

Ioannou GN. Implications of elevated serum alanine aminotransferase levels: Think outside the liver. Gastroenterology. 2008;135:1851-4.

30.

Campos F, Rodriguez-Yanez M, Castellanos M, Arias S, Perez-Mato M, Sobrino T, et al. Blood levels of glutamate oxaloacetate transaminase are more strongly associated with good outcome in acute ischaemic stroke than glutamate pyruvate transaminase levels. Clin Sci (Lond). 2011;121:11-7. 31 .

Campos F, Sobrino T, Ramos-Cabrer P, Castellanos M, Blanco M, Rodriguez-Yanez M, et al. High blood glutamate oxaloacetate transaminase levels are associated with good functional outcome in acute 
ischemic stroke. J Cereb Blood Flow Metab. 2011;31:1387-93.

\section{Figures}

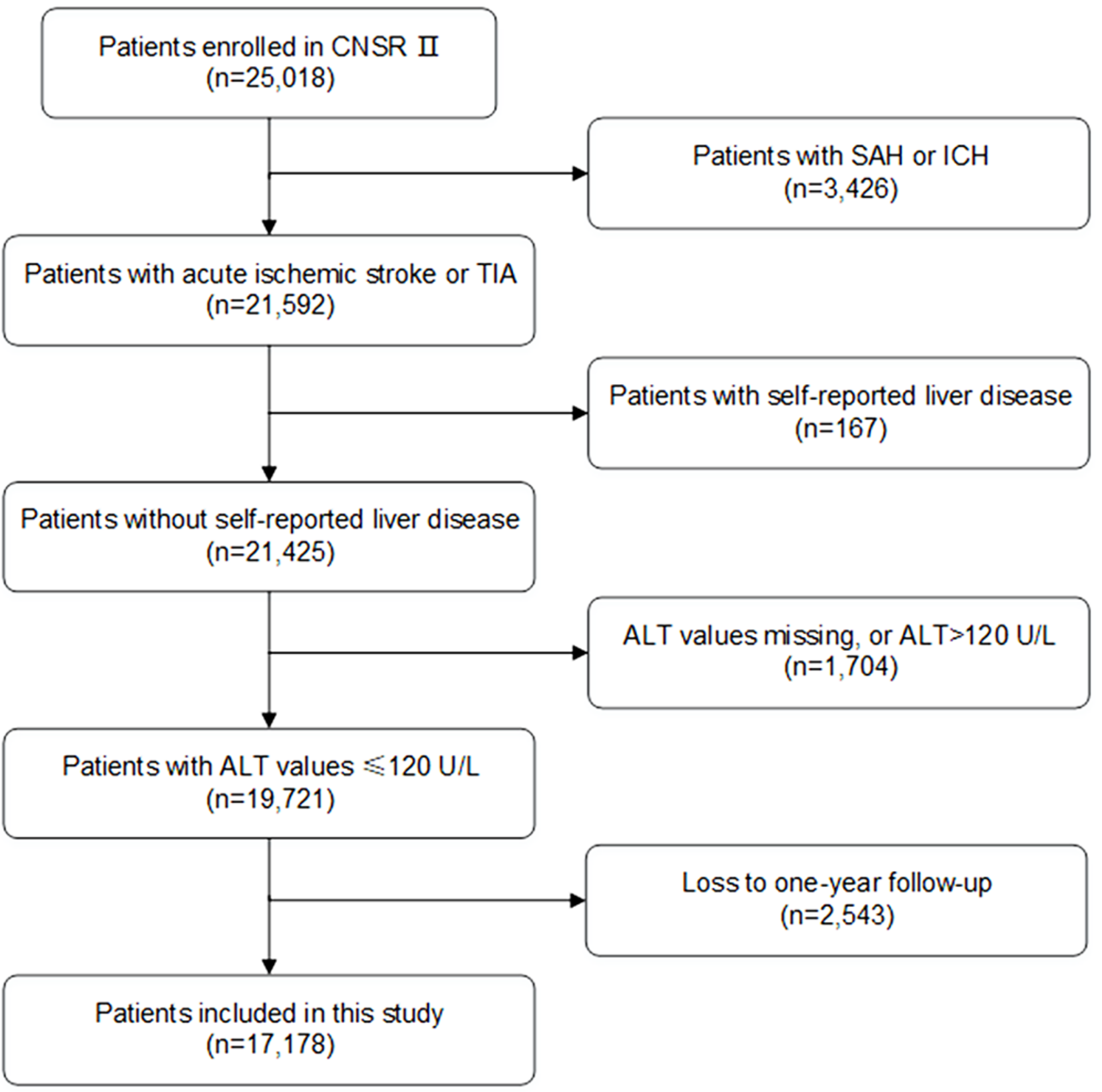

Figure 1

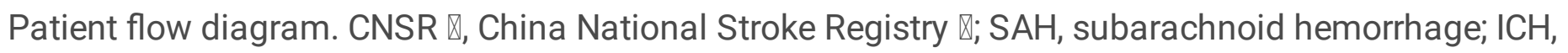
intracerebral hemorrhage; TIA, transient ischemic attack; ALT, alanine aminotransferase. 


\begin{tabular}{|c|c|c|c|c|}
\hline Outcomes & Crude OR $(95 \% \mathrm{Cl})$ & Adjusted OR (95\% Cl) & & P for trend \\
\hline All-cause mortality & & & & $<0.0001$ \\
\hline Q2 vs. Q1 & $0.63(0.54-0.74)$ & $0.71(0.58-0.87)$ & $\longmapsto$ & \\
\hline Q3 vs. Q1 & $0.70(0.60-0.82)$ & $0.83(0.67-1.01)$ & $\longmapsto$ & \\
\hline Q4 vs. Q1 & $0.59(0.51-0.70)$ & $0.70(0.56-0.87)$ & $\longmapsto$ & \\
\hline Q5 vs. Q1 & $0.57(0.49-0.68)$ & $0.55(0.43-0.70)$ & $\longmapsto$ & \\
\hline Recurrent stroke & & & & 0.006 \\
\hline Q2 vs. Q1 & $0.67(0.53-0.85)$ & $0.77(0.59-0.99)$ & $\longmapsto$ & \\
\hline Q3 vs. Q1 & $0.81(0.65-1.00)$ & $0.91(0.71-1.16)$ & $\longmapsto$ & $\longrightarrow$ \\
\hline Q4 vs. Q1 & $0.66(0.53-0.83)$ & $0.74(0.56-0.97)$ & $\longmapsto$ & \\
\hline Q5 vs. Q1 & $0.59(0.46-0.74)$ & $0.61(0.45-0.83)$ & $\longmapsto$ & \\
\hline Composite endpoint & & & & 0.0001 \\
\hline Q2 vs. Q1 & $0.69(0.59-0.80)$ & $0.79(0.66-0.94)$ & $\mapsto$ & \\
\hline Q3 vs. Q1 & $0.74(0.64-0.86)$ & $0.87(0.73-1.04)$ & $\longmapsto$ & H \\
\hline Q4 vs. Q1 & $0.62(0.54-0.73)$ & $0.73(0.60-0.89)$ & $\longmapsto$ & \\
\hline Q5 vs. Q1 & $0.62(0.53-0.72)$ & $0.62(0.49-0.77)$ & $\mapsto$ & \\
\hline Poor functional outcome & & & & 0.0005 \\
\hline Q2 vs. Q1 & $0.65(0.58-0.72)$ & $0.74(0.64-0.86)$ & $\mapsto-1$ & \\
\hline Q3 vs. Q1 & $0.69(0.62-0.77)$ & $0.86(0.74-1.01)$ & 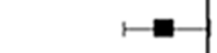 & \\
\hline Q4 vs. Q1 & $0.61(0.54-0.68)$ & $0.79(0.67-0.93)$ & $\mapsto-1$ & \\
\hline Q5 vs. Q1 & $0.55(0.49-0.62)$ & $0.67(0.56-0.80)$ & $\mapsto$ & \\
\hline & & & 0.50 & 1.50 \\
\hline
\end{tabular}

Figure 2

Crude and adjusted odds ratios of ALT levels for one-year adverse outcomes. In multivariable analysis, variables adjusted including age, gender, diabetes, hypertension, dyslipidemia, history of stroke, coronary heart disease, atrial fibrillation, current or previous smoking, moderate or heavy alcohol, baseline National Institutes of Health Stroke Scale (NIHSS), mRS at discharge, body mass index (BMI), AST levels, white blood cell count, antihypertensive drugs, lipid-lowering drugs, hypoglycemia drugs and pneumonia during hospitalization. OR, odds ratio; $\mathrm{Cl}$, confidence interval; Q, quintile. 


\section{A. All-cause mortality}

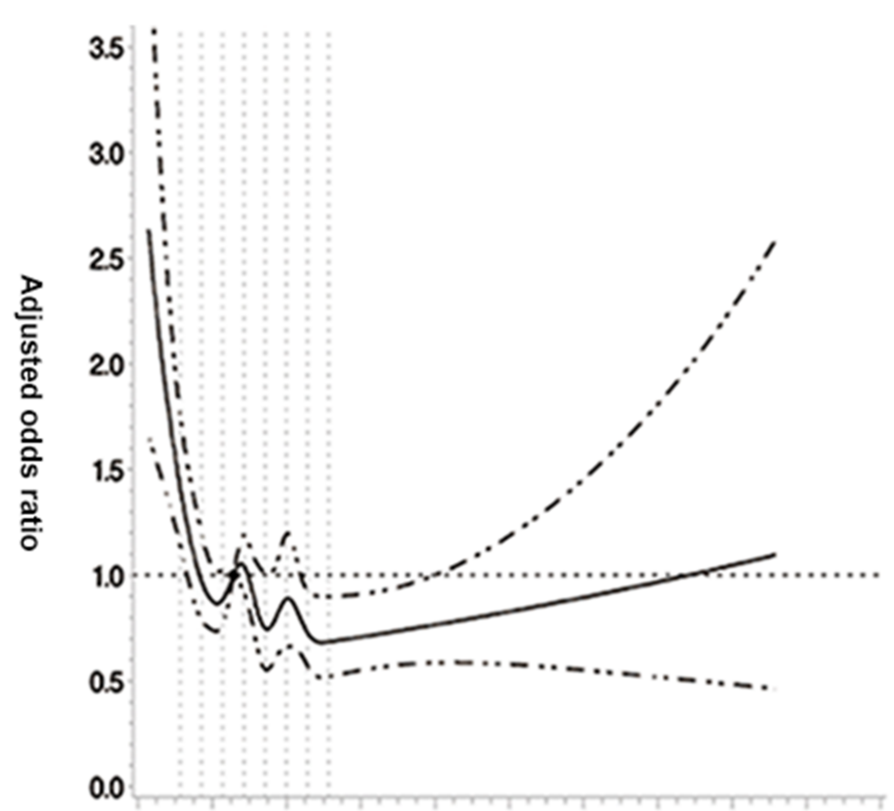

C. Composite endpoint

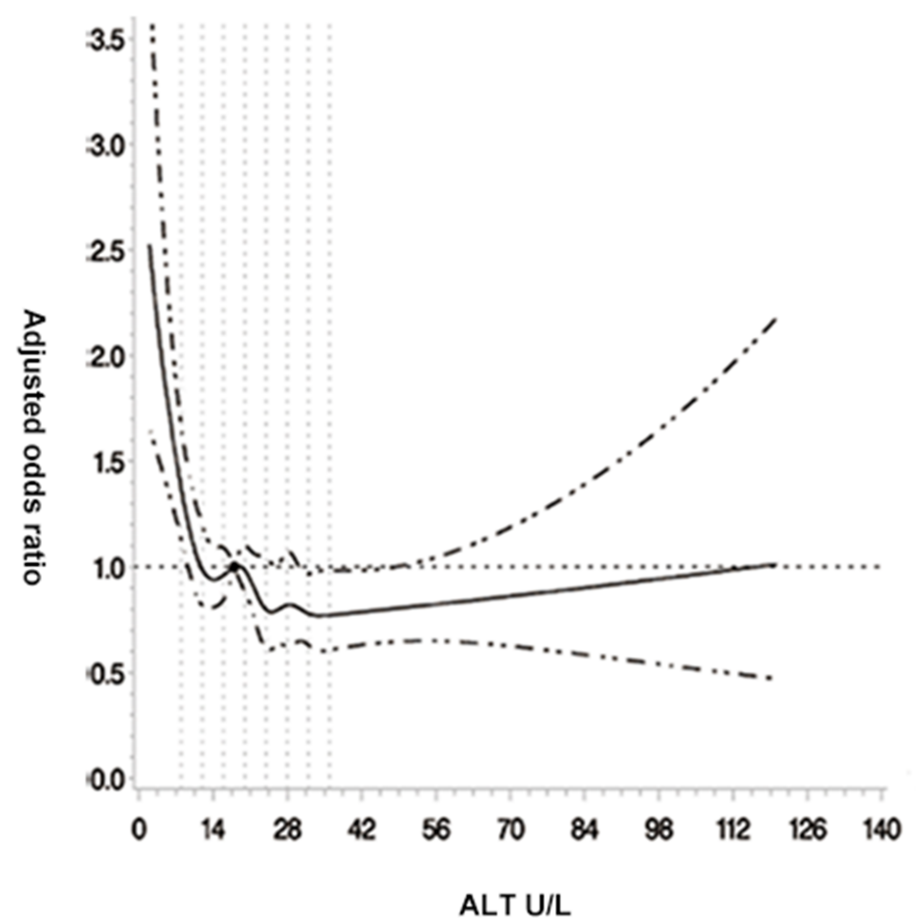

B. Recurrent stroke

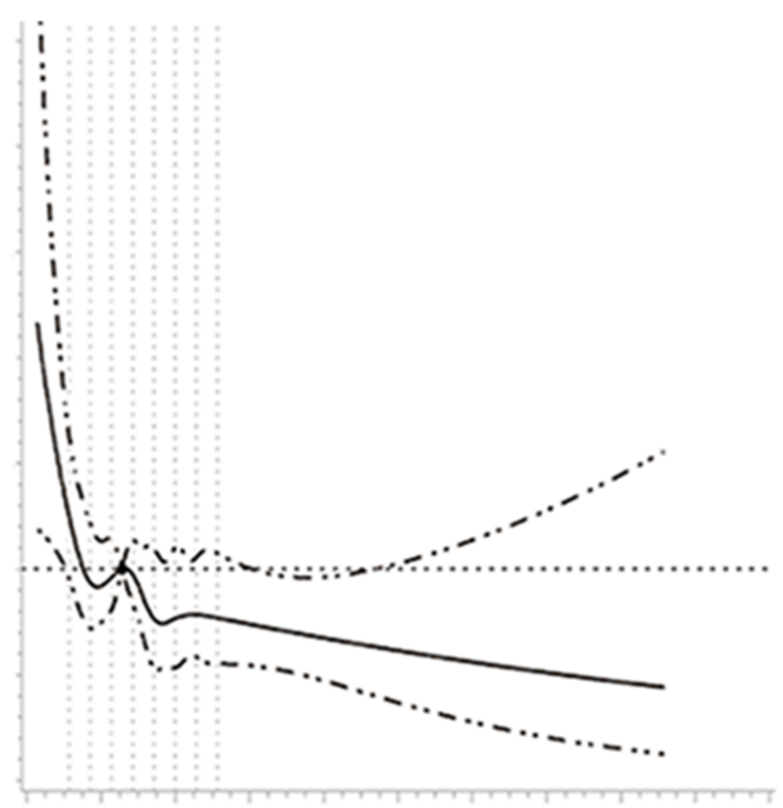

D. Poor functional outcome

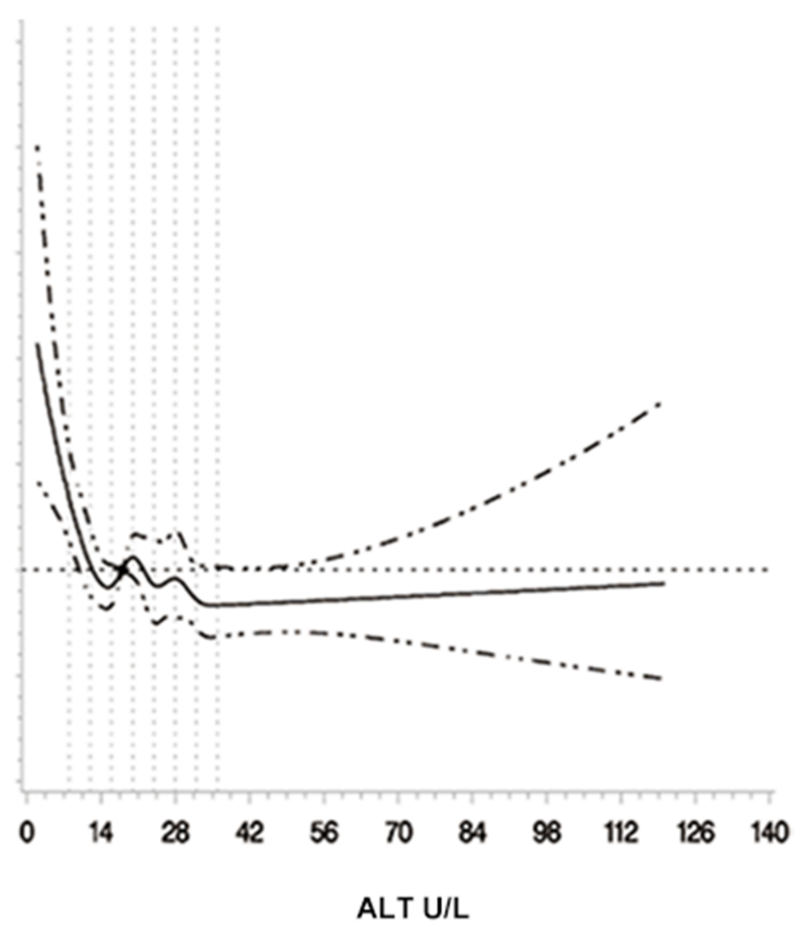

\section{Figure 3}

The patterns of associations between ALT levels and adverse stroke outcomes. (A) All-cause mortality; (B) Recurrent stroke; (C) Composite endpoint; (D) Poor functional outcome. The solid line indicates adjusted odds ratio and the dashed lines the $95 \%$ confidence interval bands. Odds ratios were obtained by restricted cubic spline logistic regression after adjustment for confounding factors, with knots placed 
at $8,12,16,20,24,28,32$ and $36 \mathrm{U} / \mathrm{L}$ of ALT levels and the median serum ALT value (18 U/L) as the reference.

\section{Supplementary Files}

This is a list of supplementary files associated with this preprint. Click to download.

- Additonalfile2.docx

- Additionalfile1.docx 